\title{
A Dynamic Model of the Eye Nystagmus Response to High
}

\section{Magnetic Fields}

\section{Paul M Glover ${ }^{1}$, Yan Li ${ }^{1}$, Andre Antunes ${ }^{1}$, Omar S Mian ${ }^{2}$ and Brian L Day ${ }^{2}$}

${ }^{1}$ The Sir Peter Mansfield Magnetic Resonance Centre, University of Nottingham,

Nottingham, NG7 2RD, UK

${ }^{2}$ Sobell Department of Motor Neuroscience and Movement Disorders, Institute of Neurology,

University College London, London, WC1N 3BG, UK

\section{Running Title:}

Eye Nystagmus Dynamics in a Magnetic Field

\section{Contact Information:}

Dr Paul Glover

The Sir Peter Mansfield Magnetic Resonance Centre

School of Physics and Astronomy

University of Nottingham

Nottingham

NG7 2RD

Tel: +44 (0) 1158466379

Fax: +44 (0) 1159515166

email: Paul.Glover@Nottingham.ac.uk 


\section{Abstract}

It was recently shown that high magnetic fields evoke nystagmus in human subjects with functioning vestibular systems. The proposed mechanism involves interaction between ionic currents in the endolymph of the vestibular labyrinth and the static magnetic field. This results in a Lorentz force that causes endolymph flow to deflect the cupulae of the semi-circular canals to evoke a vestibular-ocular reflex (VOR). This should be analogous to stimulation by angular acceleration or caloric irrigation. We made measurements of nystagmus slow-phase velocities in healthy adults experiencing variable magnetic field profiles of up to 7 Tesla while supine on a bed that could be moved smoothly into the bore of an MRI machine. The horizontal slow-phase velocity data were reliably modelled by a linear transfer function incorporating a low-pass term and a high-pass adaptation term. The adaptation time constant was estimated at $39.3 \mathrm{~s}$ from long-exposure trials. When constrained to this value, the lowpass time constant was estimated at $13.6 \pm 3.6 \mathrm{~s}$ (to $95 \%$ confidence) from both short and long exposure trials. <text removed about velocity storage time constant > This confidence interval overlaps with values obtained previously using angular acceleration and caloric stimulation. Hence it is compatible with endolymph flow causing a cupular deflection and therefore supports the hypothesis that the Lorentz force is a likely transduction mechanism of the magnetic-field evoked VOR.

\section{Keywords:}

Magnetic fields; bio-magnetic effects; vestibular-ocular reflex.

\section{PACS: $87.85 . T u$}




\section{Introduction}

High-field magnetic resonance imaging (MRI) is increasingly used for research and clinical purposes due to the benefits of potentially high spatial resolution, signal to noise and contrast to noise ratios. It has been appreciated for some time that motion perception (or vertigo-like sensation) is sometimes reported by some subjects and workers in and around the high-field ( $\geq 3$ tesla) MRI scanners (Schenck, 1992; Liu et al., 2003; Gowland, 2005; Theysohn et al., 2008; Heilmaier et al., 2011). Recently, Roberts et al., (2011) put forward a novel mechanism that could form part of the explanation for these perceptual effects. They demonstrated that static magnetic fields evoke a robust nystagmus that is contingent on a functioning vestibular system. The response is long-lasting and persists while the head is stationary in a spatially homogenous magnetic field with characteristics typical of continuous stimulation of semicircular canals. A number of mechanisms by which magnetic fields may stimulate the vestibular system had previously been proposed Glover et al., (2007), but they require movement through magnetic fields, time-varying magnetic fields or inhomogeneous magnetic fields. These requirements excluded them as the principle cause of the nystagmus observed by Roberts et al., (2011). It was instead proposed that static magnetic fields could stimulate the semi-circular canals through interaction with naturally occurring ionic currents in the endolymph fluid resulting in a Lorentz force. The hair cells of the vestibular system have a significant resting state current of order $80 \mathrm{pA}$ per unit, mainly due to a net potassium ion flux (Marcus et al., 1994). Overall currents flowing between the dark cells of the epithelium to the hair cells on the crista in the human ampulla can be estimated at $700 \mathrm{nA}$. Hence, such a nondiffusive ionic flux will generate a Lorentz force within the fluid. It might also be expected that such a Lorentz force would cancel out due to the circulating nature of the current. However, due to the non-symmetric geometry of the ampulla and taking the actual current paths into account, a significant residual force (leading to a net cupula pressure) will be generated. The magnitude of the current flux, the vestibular geometry and the pressures 
generated are discussed in more detail by both Roberts et al., (2011) and Antunes et al., (2012). Changes in direction and magnitude of the resulting vestibular-ocular reflex (VOR) with orientation of the head were consistent with changes in the direction and magnitude of the Lorentz force, estimated using a simple model (Roberts et al., 2011). A more realistic and complex model that took account of labyrinth geometry, fluid dynamics and ion flow direction was described by Antunes et al., (2012) supporting the notion that the Lorentz forces were indeed strong enough to cause vestibular stimulation.

It is generally accepted that semi-circular canal mechanical response can be modelled by a simple second order transfer function. This can be derived from an inertial force due to angular acceleration balancing both the friction from a viscous fluid together with a cupula deflection spring constant (van Egmond et al., 1949; Oman et al., 1987; Oman and Young, 1972; Fernandez and Goldberg, 1971). The hair cell afferent firing rate has been shown to have a linear response to cupula deflection within the normal operating range resulting in a transducer which effectively senses angular velocity for normal movements of the head. Angular velocity is coupled to the eyes via a neural route which contains additional dynamic processes. If the Lorentz force mechanism is responsible for transduction of the magnetic stimulation then the eye nystagmus response should behave in the same way as it does to constant acceleration stimuli.

In this work we aim to model the dynamics of the magnetic field-evoked VOR in human subjects experiencing magnetic field strengths of up to 7 Tesla. The model can then be compared to that expected from the hypothesised mechanism. It is important to fully understand the mechanisms of any bio-effect of magnetic fields as both users and regulatory bodies wish to minimise any possibility of long-term health risks (Ziegelberger, 2009b, a). Such knowledge will be invaluable in the debate about high magnetic fields in the workplace 
and likelihood of long or short term exposure effects. It also opens up the possibility of using magnetic fields as a novel mode of vestibular stimulation for research and clinical purposes.

\section{Model}

The transfer function model proposed in this work is shown in diagrammatic form in Figure 1 with components representing endolymph-cupular transduction as well as adaptation to vestibular input. It is equivalent to models used to characterise the nystagmus slow phase velocity response to continuous angular acceleration (Young and Oman, 1969; Malcolm and Jones, 1970) and caloric (Formby and Robinson, 2000; Tsutsumi et al., 2011) stimulation of the semi-circular canals. The cupula deflection model is based on the response of a simple damped second order differential equation. An angular acceleration provides an inertial force proportional to the mass of fluid. Viscous forces and the spring constant of the cupula determine the time-constants of the system. These may be decomposed into short, $\tau_{S}$, and long, $\tau_{L}$, time-constants and may be related to the viscosity and density of the endolymph as well as the geometry of the semi-circular canals (van Egmond et al., 1949; Oman et al., 1987). Most authors describe experimental measurements of, or numerical calculations for these time constants as being in the range of a few ms for $\tau_{S}$ and of the order of $10 \mathrm{~s}$ for $\tau_{L}$. (Oman et al., 1987) shows that for normal head movement frequencies the transfer function simplifies to that of an integrator $\left(1 / i \omega \tau_{L}\right)$, thus the cupula response becomes proportional to angular velocity. The gain of the system for a magnetic input can be calculated and compared to inertial inputs (Antunes et al., 2012). However, in the model described here the eye nystagmus slow-phase velocity (SPV) gain is simply replaced by $k_{l}\left(\right.$ degrees $\mathrm{s}^{-1} \mathrm{~T}^{-1}$ ).

The adaptation part of the process has a time constant, $\tau_{A}$, of the order of a minute. This process includes a potential peripheral mechanism (Fernandez and Goldberg, 1971) as well as 
a central mechanism (St George et al., 2011). In our model an adaptation gain, $k_{2}$, is included which allows for only a proportion of the response to be removed. Without this term the SPV would decline to zero, which was not apparent in the magnetic field stimulation data of (Roberts et al., 2011). Hence the combined transfer function relating eye SPV response ( $S P V(s))$ due to a magnetic field input $(B(s))$ may be written as,

$$
\frac{S P V(s)}{B(s)}=\frac{k_{1}}{\left(1+s \tau_{S}\right)\left(1+s \tau_{L}\right)} \frac{\left(1-k_{2}\right)+s \tau_{A}}{\left(1+s \tau_{A}\right)}, \quad \text { Eq. } 1
$$

where the symbol ' $s$ ' is the Laplace variable derived from the Laplace transform (in which the differential operator $d / d t \rightarrow s \rightarrow i \omega)$. The temporal response model was implemented as a digital filter in MATLAB. A four parameter model was used initially based on $\tau_{L}, \tau_{A}, k_{l}$ and $k_{2}$. The short time constant, $\tau_{S}$, was fixed to $5 \mathrm{~ms}$ as it has no effect on the temporal response at the low stimulus frequencies of the current experiment.

It is useful to consider the changes to the model should the magnetic field input to the eye SPV response have a different origin. If the rate of change of magnetic field is acting as an input (i.e. an induced electric field) then this effectively would introduce an extra zero into the transfer function described above. It would not then be possible for there to be a long-term response to a static field without a neural-based integrator, effectively a pole which cancels out the zero. Such an integrator, if present, would be observable as an increasing response during long angular acceleration step changes, which does not occur. In addition, any susceptibility related mechanism is likely to only have an input at the entrance to the bore of the magnet where spatial gradients are at their maximum (Glover et al., 2007). The input profile would also be an impulse or transient event. However, susceptibility mechanisms would not have a component proportional to the sign of the rate of change of magnetic field (as for induced current), but be unipolar (i.e. non-linear) in nature. Again, as with induced current mechanisms, an integration term would be required to generate a long term response. 
It is possible that a combination of mechanisms are present, if so we would expect any SPV response would not then fit a simple linear transfer function model.

\section{Materials and Methods}

Seventeen healthy subjects (10 male, 7 female, 20 - 32 yr old) participated in this study. The data shown in this paper were extracted from a larger series of tests involving multiple sessions. Individual trials reported here are coded in the form $\mathrm{S}<$ subject number $\rangle_{-}<$session>_<trial>. Eleven of these took part in session 1 (called the short-entry study) with 6 males and average age 25 years. Nine subjects took part in sessions 3 and 4 (called long-entry studies) with 6 males and average age 26 years. Three subjects appear in both cohorts and some subjects have more than one trial in a session. No subjects had a history of any hearing disorder or conditions which affected their inner ear. The written consent of all subjects was obtained after a full explanation of the experimental procedure. The experimental protocol was approved by the Medical School Research Ethics Committee of the University of Nottingham.

Experiments were carried out on a Philips Healthcare Achieva 7.0 T MRI scanner (Cleveland OH, USA) which has a (non-actively shielded) magnet originally supplied by Magnex (Yarnton UK, now Agilent). No imaging scan was performed (which would involve switched and RF magnetic fields) so only the static magnetic field (B) was present. The direction of B was axial with the bore and from head to toe when the subject entered the magnet head first. The experiment setup is shown in Figure 2. A custom-made patient bed and track was installed to replace the original Philips bed. This replacement bed moves smoothly on 100 $\mathrm{mm}$ rubber tyres so that the subject is unable to discern any linear motion, thus minimising any motion cue. The track extended 8 meters in total length from the service (rear) end of the scanner to the entrance of the scanner room. This long track meant the baseline magnetic field 
exposure of the head at the start of, and between, trials was considerably lower $(<0.1 \mathrm{~T})$ than if the standard Philips bed had been used $(\sim 0.7 \mathrm{~T})$. This low value is desirable to reduce possible baseline effects of the field.

An infra-red (IR) camera was positioned above one of the subject's eyes (usually left) to record the eye movement in the dark. This camera was an adapted webcam (V-U0012 Logitech) where the components had been removed and placed in a plastic box which was mounted on a frame with a flexible 'gooseneck' fitting. The lens barrel of the camera was temporarily removed and the IR-cut filter removed. This extended the camera's sensitivity into the near IR. Two HIRL5040 IR (920nm wavelength) LEDs were used for illumination carrying $30 \mathrm{~mA}$ current each. These LEDs were chosen for their narrow spectral output as many IR LEDs have visible (to some subjects) red components. The subjects wore a pair of modified glasses throughout the video recording of the eye nystagmus. The glasses didn't have lenses, but had large black-and-white rectangular frames, which provided a reference frame for the pupil tracking. The scanner room was made as dark as possible. Additionally, the subjects' head and the infrared camera were covered by a layer of black felt cloth so that it was completely dark and no visual cues could be obtained. It is desirable to obtain the VOR without confound from visual and motion cues. The removal of cues was effective as subjects had difficulty explaining if they were being pushed or stationary.

Two additional unmodified webcams (V-U0012 Logitech) were fixed to the wall beside and above the bed to take a picture of the subjects' initial head location before the experiment. Figure 3 shows photographs (taken by these datum cameras) of subjects on the bed at the start position. These cameras were used to record the subject's Reid's plane and head roll angles relative to the laboratory frame of reference (fig 3B). In addition the cameras measured the 
orientation and position of the IR camera and glasses frames in order to transform the video recording coordinates into those of the subject's frame of reference.

An optical encoder (HEDS-5701-F00, Hewlett Packard) was driven by one of the wheels of the movable patient bed in order to record the displacement and speed of the bed. The data acquisition was controlled on a PC using an interactive program written in MATLAB (MathWorks Inc.) version 7.10 through a data acquisition (NI-DAQ ${ }^{\mathrm{TM}} \mathrm{mx}$, USB-6009, National Instruments) device. An audible beep of one second was generated by the interactive MATLAB program at the same time as the start of the encoder data acquisition, and picked up through a bed-mounted microphone which interfaced with the eye camera recording software. The purpose of the beep was to synchronise the video and position recordings which enabled determination of the relationship between the magnetic field and recorded eye movements.

At the start of each trial, the subject would lay still in the supine position, with their head nearer the bore than their feet (head-first entry), head pitch angle close to neutral (Reid's plane vertical) and head roll angle zero degree relative to the direction of the main magnetic field $\mathrm{B}_{0}$. Subjects were instructed to keep their eyes open throughout each trial. After positioning pictures had been recorded the lights were switched off and the subject covered to exclude any remaining light. At this point the video and position recordings were started. A stopwatch was used to control the speed of bed movement and duration of field exposure. During each trial, the subjects were manually pushed by an experimenter slowly towards the scanner taking about $50 \mathrm{~s}$ to reach $7 \mathrm{~T}$. At this point two different protocols were followed:

A) For the short-entry protocol the subjects only stayed in the magnet bore until they indicated verbally that they had perceived a sensation of motion (which, on average, occurred at around $5 \mathrm{~T}$ so the subject's head did not always reach the full $7 \mathrm{~T}$ 
available). The subjects were then removed slowly from the field back to the initial position. There was then a wait period of between 30 and $50 \mathrm{~s}$ and the procedure was repeated a further 3 times. No practice trial was given for this protocol.

B) For the long-entry protocol the subjects stayed in the scanner bore at the maximum magnetic field for about $135 \mathrm{~s}$ then were pulled out slowly back to the initial position. After a period which varied between 30 and $60 \mathrm{~s}$ the recordings were halted and the lights were switched on. A practice trial was given to each subject for them to be familiar with the experience.

All recorded data were post processed. The initial location of the vestibular system (approximated from the external auditory meatus) relative to a room-fixed reference was calculated from the still pictures. The displacement and velocity of the vestibular system were calculated from the collected encoder data using a MATLAB routine. By interpolating the field value for the location of the vestibular system using the magnetic field profile of the magnet provided by Philips, we obtained the value for the magnetic field the vestibular system experienced with time. The resolution of the bed position measurement was better than $1 \mathrm{~mm}$. The position of the vestibular organ relative to the magnetic field depends on the camera precision, slight subject movements and a precise knowledge of the vestibular system relative to the auricular canal. Hence a conservative estimate of total position uncertainty was taken as $\pm 20 \mathrm{~mm}$. Even during the very highest rate of change of field this uncertainty could only give an error of 0.1 tesla in the calculated magnetic field profile. If the start and finish positions are well defined (0.098 to 7.0 tesla) then this error is not propagated. In addition, even at the slow speed of the bed, this magnetic field uncertainty might only account for a temporal error of 0.5 seconds at the maximum rate of change. 
Automated pupil tracking was performed offline using in-house written software. The algorithm consisted of measuring the centre of the pupil relative to the coordinate frame provided by the glasses. This was achieved by calculating the average centre of ellipses constructed from points in the high contrast border between the pupil and iris. A visual verification of the quality of this fit was made, giving smooth and consistent results. No eye movement data were obtained in the video frames where the subject's eye was closed due to blinking or the majority part of the eye pupil was covered by the eyelid or eyelashes. Pupil displacements were transformed from glasses to cranial coordinates (horizontal axis $=$ interaural axis; vertical axis perpendicular to Reid's plane) and then converted to eye rotations using simple trigonometry based on the distance between camera and the pupil, eye size and eye displacement. There were some approximations and errors associated to this calculation distance from eye to camera is considered constant; eye displacement is considered to occur in the plane orthogonal to the camera; and the assumption of a $12 \mathrm{~mm}$ eyeball radius, which is known to have little variation between adults (s.d., $0.08 \mathrm{~mm}$; (Jansson, 1963)). A conservative figure for the resultant of the errors of the eye angular velocity is $10 \%$. The slow-phase velocity was then determined from the slow drift of the eye angle between nystagmus fast phases.

The parameter fitting is performed in MATLAB using the non-linear least squares method based on the Levenberg-Marquardt (LM) algorithm. The model transfer function of equation 1 is implemented using a digital filter with coefficients calculated using a bi-linear ztransform of the numerator and denominator polynomials. The temporal magnetic field profile is filtered to give the predicted SPV response. The LM algorithm adjusts either 3 or 4 chosen input parameters to give a minimum mean-square-error (MSE) between the data and the predicted response. The best estimate of the parameters is returned by the LM algorithm together with a co-variance matrix which yields the confidence interval (calculated to 95\%). 
Even though SPV could be observed on the video recordings it was not always possible to quantify the SPV for the entire record. Subjects tended to let their eyes close, particularly towards the end of the recording. We have established a number of inclusion criteria for both protocols. For the long-entry protocol these are:

a) Peak response of SPV exceeding 5 degrees/s. It was felt that signal-to-noise ratio on data sets that met this criteria would be adequate for reliable fits.

b) Periods of SPV data lasting at least 20s and commencing during the transition between 0T and 7T (entry) or the transition between 7T and 0T (exit). Data in one or both of these periods are required for estimation of $\tau_{L}$.

c) At least 100s of SPV data during the period of stable exposure 7T. This is required for estimation of $\tau_{A}$.

Trials are included in a four parameter fit if all 3 criteria are met. Trials are included in a three parameter (TA fixed) fit if criteria a and $\mathrm{b}$ are met. For the short-entry protocol the inclusion criteria are:

a) Peak response of SPV exceeding 5 degrees/s.

b) SPV data for the duration of at least two entry periods

c) SPV data points during at least one of the out-of-magnet periods between entry periods. This is required to fit the $k_{2}$ parameter.

In short-entry trials, the exposure and exit periods are of order $\tau_{A}$ so a 4 parameter fit is not possible. Short-entry trials are only included in a three parameter fit if all three criteria are met.

\section{Results}

The subjects' head positions had mean pitch and roll angles of $12 \pm 5$ degrees and $2 \pm 3$ degrees respectively. Positive pitch angles denote flexion and positive roll indicates right ear to shoulder. The average velocity of the bed during entry into the scanner bore was 
approximately $0.07 \mathrm{~m} \mathrm{~s}^{-1}$. The low bed speed used results in a typical maximum rate of change of field strength of about $0.6 \mathrm{~T} \mathrm{~s}^{-1}$ during movement through the static magnetic field gradient at the entrance to the $7 \mathrm{~T}$ magnet bore. Note that the speed of the bed used for this experiment was slower than would be experienced by a subject lying on the original Philips bed whilst having an MRI scan (typically the bed velocity is about $0.13 \mathrm{~m} \mathrm{~s}^{-1}$ ).

All 17 subjects developed leftwards SPV nystagmus during the trials. Some subjects also developed a slight vertical component of SPV nystagmus but this was of variable direction and hence only horizontal components have been modelled in the current study. A typical processed recording of SPV and the magnetic field profile is shown in both Figure 4 (Subject S023) and Figure 5 (Subject S004). The delayed rise and the long adaptation time can be observed. The SPV does not return to zero even after more than two minutes exposure to the field and reverses upon withdrawal from the field. This confirms observations of Roberts et al., (2011).

Six of the 12 long-entry trials conducted had SPV data which met the inclusion criteria for an unconstrained 4 parameter fit and these are summarised in Table 1. Condition (a) is not met in S024_4_3, S010_3_1 and S010_3_7. Condition (b) is not met in S023_4_2 and condition (c) is not met in S009_4_6 and S012_3_2. In all tables the individual trial parameter fit uncertainties are given to a $95 \%$ confidence level (i.e. mean value \pm 2 times Standard Error of the Mean). A significant issue in attempting to fit using 4 degrees of freedom was the sensitivity to the adaptation parameter, $\tau_{A}$. To improve the fit confidence, and to be able to drop inclusion criteria (c), we constrained $\tau_{A}$ to the mean value obtained from the fits reported in Table $1(39.3 \mathrm{~s})$. Constraining $\tau_{A}$ to a particular value will have a small effect on the remaining parameters and this can be seen in Table 2 but the uncertainty in the 3 remaining fitted parameters reduces significantly demonstrating a greater reliability. In addition the MSE 
reported from both 3 and 4 parameter fits for the same trial remains the same indicating that the fit has not been degraded by the additional constraint. In Table 2 subject S012 now has multiple trials included in the 10 listed. In these cases trial values are averaged together first as before.

Choosing an arbitrary value of $120 \mathrm{~s}$ for $\tau_{L}$ (which is at the extreme end of the range as reported in the literature) instead of $39.3 \mathrm{~s}$ and running the fit again for the whole data set results in a systematic reduction of approximately $4.0 \mathrm{~s}$ in the mean value for $\tau_{L}$ (within the $95 \%$ confidence interval for this parameter). However, it is quite clear that both from visual inspection, and from the calculated value of MSE from each attempted fit, that such a high value of adaptation parameter does not match the measured SPV for these subjects (this data is not tabulated or shown here).

Figure 5 demonstrates the effect of removing the cupula deflection model from the fit process, i.e. only $k_{1}$ and $k_{2}$ are used with $\tau_{A}$ constrained at $39.3 \mathrm{~s}$. In general, removing the cupula deflection component from the model produces an overshoot of the initial response and it is clear that the delay caused by such a low-pass section is required to characterise fully the SPV response. The MSE of the fit (given in Table 2 for comparison) with cupula deflection part of the model removed are, on average $70 \%$ higher than with $\tau_{L}$ in the model. MSE values used in this way can only be a guide as they scale with the amplitude of the response and quality of the trial data but relative comparisons can be made for each trial data set.

Figure 6 shows a family of SPV responses, due to an example magnetic field profile, generated using the four-parameter fit model (values from Table 1). The average response profile shown is not an average of the curves or SPV data sets but based on the average parameters computed in Table 1. It is not possible to average the measured SPV data sets 
together because of the slightly different magnetic field temporal profiles experienced by the subjects.

Figure 7 shows an SPV response for the short-entry experiment from trial S009_1_1 where the magnetic field temporal profile shows four brief excursions into the bore of the magnet. In this case only a 3-parameter can be performed with $\tau_{A}$ being set to $39.3 \mathrm{~s}$ as determined in Table 1. For these short-entry trials condition (c) is not met in S002_1_1 or S011_1_1 and are excluded. In addition subject S006 had to be excluded because they had a permanent natural SPV of -3 degrees/s at zero field which gave a baseline offset in the SPV data. Although their SPV had a similar overall response to the magnetic field like the other subjects, the model transfer function could not accommodate such an offset. Table 3 gives a mean value for $\tau_{L}$ of $13.5 \pm 2.9 \mathrm{~s}$ (at $95 \%$ confidence) for 8 subjects. This value is consistent with the value of $13.7 \pm 7.4$ s obtained from the 3 parameter long-entry fits in Table 2 . Combining the averages of both long-entry and short-entry experiments gives an estimate for $\tau_{L}$ of $13.6 \pm 3.6 \mathrm{~s}$.

\section{Discussion and Conclusions}

Our experimental observations replicate the findings of Roberts et al., (2011) demonstrating that static magnetic fields can evoke nystagmus. The slow-phase velocity did not depend on the rate of change of magnetic field or magnetic spatial gradients, which ruled out induced current and magnetic susceptibility mechanisms respectively (Glover et al., 2007). The observations are compatible with the hypothesis that static magnetic fields deliver a continuous vestibular stimulus via a Lorentz-force mechanism (Roberts et al., 2011) (Antunes et al., 2012). Our results additionally show that the slow-phase velocity eye nystagmus response to a magnetic field can be modelled by a linear transfer function model. A complete 
fit required adaptation and low-pass components similar to the models used to characterise the vestibular-evoked nystagmus responses to rotational acceleration (Young and Oman, 1969; Malcolm and Jones, 1970) or caloric stimulation (Formby and Robinson, 2000; Tsutsumi et $a l ., 2011)$. This is relevant because head rotation, caloric stimulation and the hypothesised Lorentz-force mechanism all produce their vestibular effects through bulk movement of endolymph causing a deflection of the cupula. It could be expected, therefore, that the time constants of their respective transfer functions should be comparable.

The low-pass component of the model required having a time-constant of $13.6 \pm 3.6 \mathrm{~s}$. The uncertainty values for the individual trial fit parameters indicate a high level (>95\%) of confidence that such a non-zero positive value of time-constant is required in all included subjects. There was a wide range of values for the overall sensitivity to magnetic field $\left(k_{l}\right)$ across subjects but a closer agreement in the proportion of adaptation $\left(k_{2}\right)$. Figure 8 shows the frequency magnitude and phase response for the model using average model parameters taken from Table 2. The cut-off frequencies due to the long (low-pass) and adaptation (high-pass) time-constants can be seen.

The peripheral vestibular long time-constant estimated from primary afferent responses to rotation in monkeys is around $6 \mathrm{~s}$ (Fernandez and Goldberg, 1971). However, the vestibularocular reflex (VOR) to vertical axis rotational stimuli has a considerably longer time constant with values of around 16-20s estimated during acceleration steps (Young and Oman, 1969; Malcolm and Jones, 1970) or during the post-rotary decline following velocity steps (Raphan et al., 1979). This prolongation of the time constant is attributed to an additional central process referred to as the velocity storage mechanism (Raphan et al., 1979). Our VOR timeconstant of around $13 \mathrm{~s}$, therefore, suggest a central velocity storage prolongation, but possibly less than during vertical axis head rotation. Head position may be an important 
variable. The longest time constants have been obtained by rotating the head about a vertical axis. Situations that stimulate the semi-circular canals with the head off-vertical (as in the current study) tend to diminish or eliminate the velocity storage contribution to the VOR and lead to shorter time constants. For example, yaw rotation about a horizontal axis (sometimes termed barbeque rotation), or when the head is reoriented from upright to horizontal immediately following vertical axis yaw rotation, have yielded time-constants of around 7-9 s (Benson and Bodin, 1966; Benson et al., 1966; Fetter et al., 1992; Tweed et al., 1994).

Caloric VOR dynamics have been studied with step-caloric protocols. In this type of experiment, steady state thermal gradient is first established with the horizontal semi-circular canals orientated in the earth-horizontal plane which causes minimal stimulation. The head is then rapidly reoriented to a position (horizontal canals vertical) that leads to optimal convective stimulation, providing a quasi-step stimulus profile producing horizontal nystagmus in head coordinates (left-right direction). This caloric-evoked horizontal eye movement response with the horizontal canals vertical is similar to the situation inside the magnet in the current study, and the reported time-constants of 5-14s (Formby and Robinson, 2000; Peterka et al., 2004; Tsutsumi et al., 2011) overlap with our estimates

The adaptation time constant, estimated at around $40 \mathrm{~s}$ in the current study, was shorter than the 80-120s estimated from vertical-axis angular acceleration stimuli (Young and Oman, 1969; Malcolm and Jones, 1970) and the 100-150 s estimated from step-caloric stimulation (Formby and Robinson, 2000; Peterka et al., 2004; Tsutsumi et al., 2011). We do not have a clear explanation for this discrepancy although we acknowledge some limitations in our data that may be relevant. First, data appropriate for estimation of this time constant was only available from 6 participants ( 8 trials). Second, we did not ask participants to undertake a continuous mental alerting task, such as mental arithmetic, which can augment the VOR 
(Collins and Guedry, 1962). However, this limitation is also applicable to other studies that have led to estimates of adaptation time constant in excess of 100s (Tsutsumi et al., 2011). Third, and most importantly, we would point out the potential error in estimating a time constant of this order of magnitude using stimulation durations of a similar order. This limitation seems to be common to all studies. Indeed, for continuous angular acceleration stimuli the maximum trial duration is capped in practice by the maximum angular velocity the machine is capable of delivering. An advantage of static magnetic field stimulation is that trial duration is effectively limitless. We did not take advantage of this in the current study because the trials we conducted were part of a larger multi-trial experiment that precluded very long trial durations. However, the potential should be exploited in future studies.

In conclusion, if the low-pass time constant measured in this study can be attributed mainly to the cupula response then the magnetic field must act on either the cupula directly or the fluid. It is difficult to envisage a physical mechanism which can act on the cupula as there is unlikely to be a significant magnetic susceptibility difference between the fluid and the cupula, and even then this would not explain the effect in a uniform static field. Hence we must conclude that a fluid pressure induced by a Lorentz force is the most likely explanation as this acts in the same way as an inertial force, both of which act as driving forces developed within the canal endolymph.

\section{Acknowledgments}

We thank Ian Thexton and Alan Dorkes for technical support. The current affiliation for Andre Antunes is The Max Planck Institute for Biological Cybernetics, Tübingen, Germany. We thank the Engineering and Physical Sciences Research Council (UK) for their support of this project (Grant numbers EP/G062692/1 and EP/G061653/1). 


\section{References}

Antunes A, Glover P M, Li Y, Mian O S and Day B L 2012 Magnetic field effects on the vestibular system: calculation of the pressure on the cupula due to ionic current-induced Lorentz force Physics In Medicine And Biology 57 4477-87

Benson A J, Bodin C B and Bodin M A 1966 Comparison of the effect of the direction of the gravitational acceleration on post-rotational responses in yaw, pitch and roll Aerospace Med 37 889-97

Benson A J and Bodin M A 1966 Effect of orientation to the gravitational vertical on nystagmus following rotation about a horizontal axis Acta OtoLaryngologica 61 517-26

Collins W E and Guedry F E, Jr. 1962 Arousal effects and nystagmus during prolonged constant angular acceleration Acta Oto-Laryngologica 54 34962

Fernandez C and Goldberg J M 1971 Physiology of peripheral neurons innervating semicircular canals of the squirrel monkey. II. Response to sinusoidal stimulation and dynamics of peripheral vestibular system Journal Of Neurophysiology 34 661-75

Fetter M, Tweed D, Hermann W, Wohland-Braun B and Koenig E 1992 The influence of head position and head reorientation on the axis of eye rotation and the vestibular time constant during postrotatory nystagmus Exp Brain Res 91 121-8

Formby C and Robinson D A 2000 Measurement of vestibular ocular reflex (VOR) time constants with a caloric step stimulus J Vestib Res 10 25-39 
Glover P M, Cavin I, Qian W, Bowtell R and Gowland P A 2007 Magnetic-fieldinduced vertigo: A theoretical and experimental investigation Bioelectromagnetics 28 349-61

Gowland P A 2005 Present and future magnetic resonance sources of exposure to static fields Progress In Biophysics \& Molecular Biology 87 175-83

Heilmaier C, Theysohn J M, Maderwald S, Kraff O, Ladd M E and Ladd S C 2011 A Large-Scale Study on Subjective Perception of Discomfort During 7 and 1.5 T MRI Examinations Bioelectromagnetics 32 610-9

Jansson F 1963 Determination of Axis Length of Eye Roentgenologically and by Ultrasound Acta Ophthalmol 41 236-\&

Liu F, Zhao H W and Crozier S 2003 Calculation of electric fields induced by body and head motion in high-field MRI Journal Of Magnetic Resonance $16199-$ 107

Malcolm R and Jones G M 1970 A quantitative study of vestibular adaptation in humans Acta Oto-Laryngologica 70 126-35

Marcus D C, Liu J Z and Wangemann P 1994 Transepithelial Voltage and Resistance of Vestibular Dark Cell Epithelium from the Gerbil Ampulla Hearing Research 73 101-8

Oman C M, Marcus E N and Curthoys I S 1987 The Influence of Semicircular Canal Morphology on Endolymph Flow Dynamics - an Anatomically Descriptive Mathematical-Model Acta Oto-Laryngologica 103 1-13

Oman C M and Young L R 1972 Physiological Range Of Pressure Difference And Cupula Deflections In Human Semicircular Canal - Theoretical Considerations Acta Oto-Laryngologica 74 324-31

Peterka R J, Gianna-Poulin C C, Zupan L H and Merfeld D M 2004 Origin of orientation-dependent asymmetries in vestibulo-ocular reflexes evoked by caloric stimulation Journal Of Neurophysiology 92 2333-45 
Raphan T, Matsuo V and Cohen B 1979 Velocity storage in the vestibulo-ocular reflex arc (VOR) Exp Brain Res 35 229-48

Roberts D C, Marcelli V, Gillen J S, Carey J P, Della Santina C C and Zee D S 2011 MRI Magnetic Field Stimulates Rotational Sensors of the Brain Curr Biol 21 1635-40

Schenck J F 1992 Health And Physiological-Effects Of Human Exposure To WholeBody 4-Tesla Magnetic-Fields During Mri Annals Of The New York Academy Of Sciences 649 285-301

St George R J, Day B L and Fitzpatrick R C 2011 Adaptation of vestibular signals for self-motion perception Journal Of Physiology-London 589 843-53 Theysohn J M, Maderwald S, Kraff O, Moenninghoff C, Ladd M E and Ladd S C 2008 Subjective acceptance of 7 Tesla MRI for human imaging Magn Reson Mater Phy 21 63-72

Tsutsumi T, Tokumaru A, Murakami M, Kawaishi J, Chida W and Watanabe K 2011 On-off asymmetries of responses to caloric step stimuli $J$ Vestib Res $21111-5$

Tweed D, Fetter M, Sievering D, Misslisch H and Koenig E 1994 Rotational kinematics of the human vestibuloocular reflex. II. Velocity steps Journal Of Neurophysiology 72 2480-9

van Egmond A A J, Groen J J and Jongkees L B W 1949 The Mechanics of the Semicircular Canal Journal Of Physiology-London 110 1-17

Young L R and Oman C M 1969 Model for Vestibular Adaptation to Horizontal Rotation Aerospace Med $\mathbf{4 0}$ 1076-\&

Ziegelberger G 2009a Amendment To The ICNIRP "Statement On Medical Magnetic Resonance (MR) Procedures: Protection Of Patients" Health Physics 97 259-61 
Ziegelberger G 2009b ICNIRP Statement on the "Guidelines for Limiting Exposure to Time-Varying Electric, Magnetic, and Electromagnetic Fields (up to 300 GHz)" Health Physics 97 257-8 
Lorentz force to membrane deflection
Adaptation

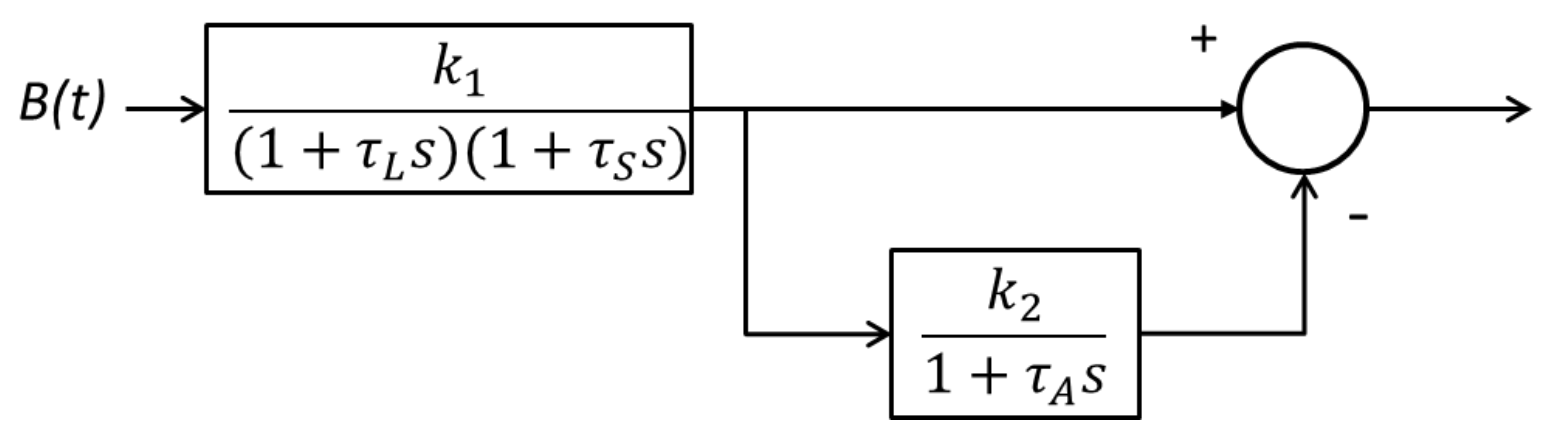

Figure 1: The transfer function representing the eye horizontal - slow phase velocity (SPV) nystagmus response to a time dependent magnetic field input.

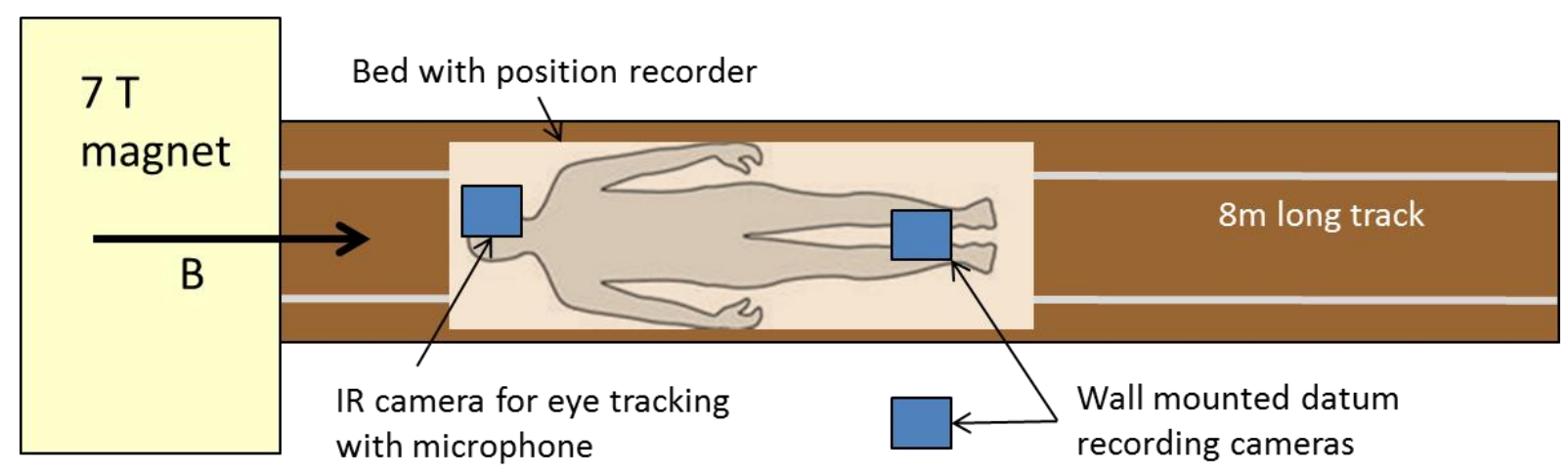

Figure 2: The experimental setup. A custom-made subject track and bed replaces the original

Philips bed. The bed is movable with six wheels along the two parallel guide tracks. The datum recording cameras were fixed relative to the track whereas the IR eye-tracking camera was fixed to the bed. 

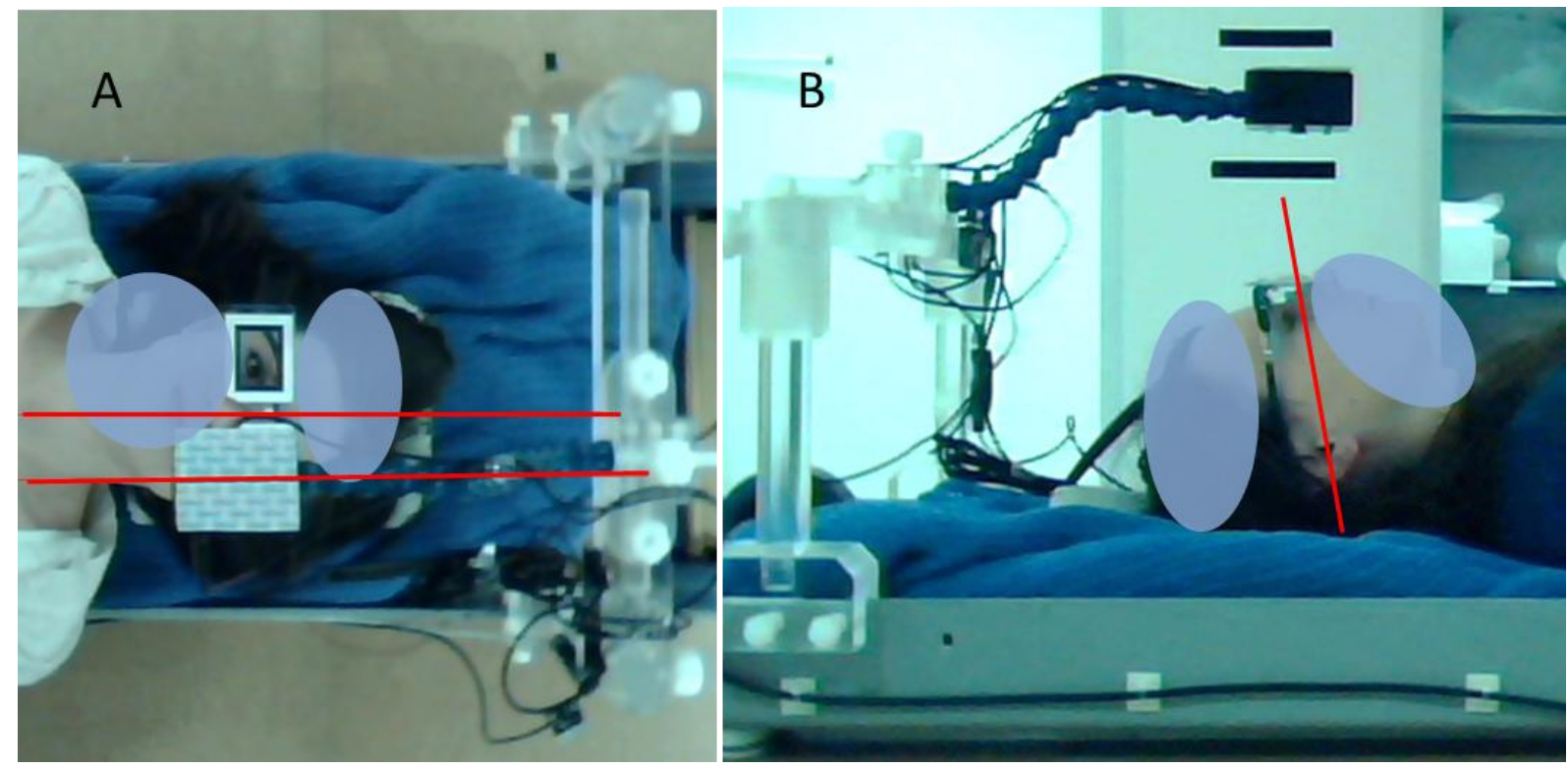

Figure 3: Photographs of the bed and two subjects taken from: A) the vertical datum camera, and B) the horizontal datum camera. The subject is lying on the bed, with the IR camera positioned above the left (recorded) eye. The far pillar and the black parallel strips are both used as a reference to calculate the initial position and angle of the vestibular system in the laboratory frame of reference. The annotated lines represent both the IR camera alignment and the head position and orientation in the laboratory frame-of reference. 


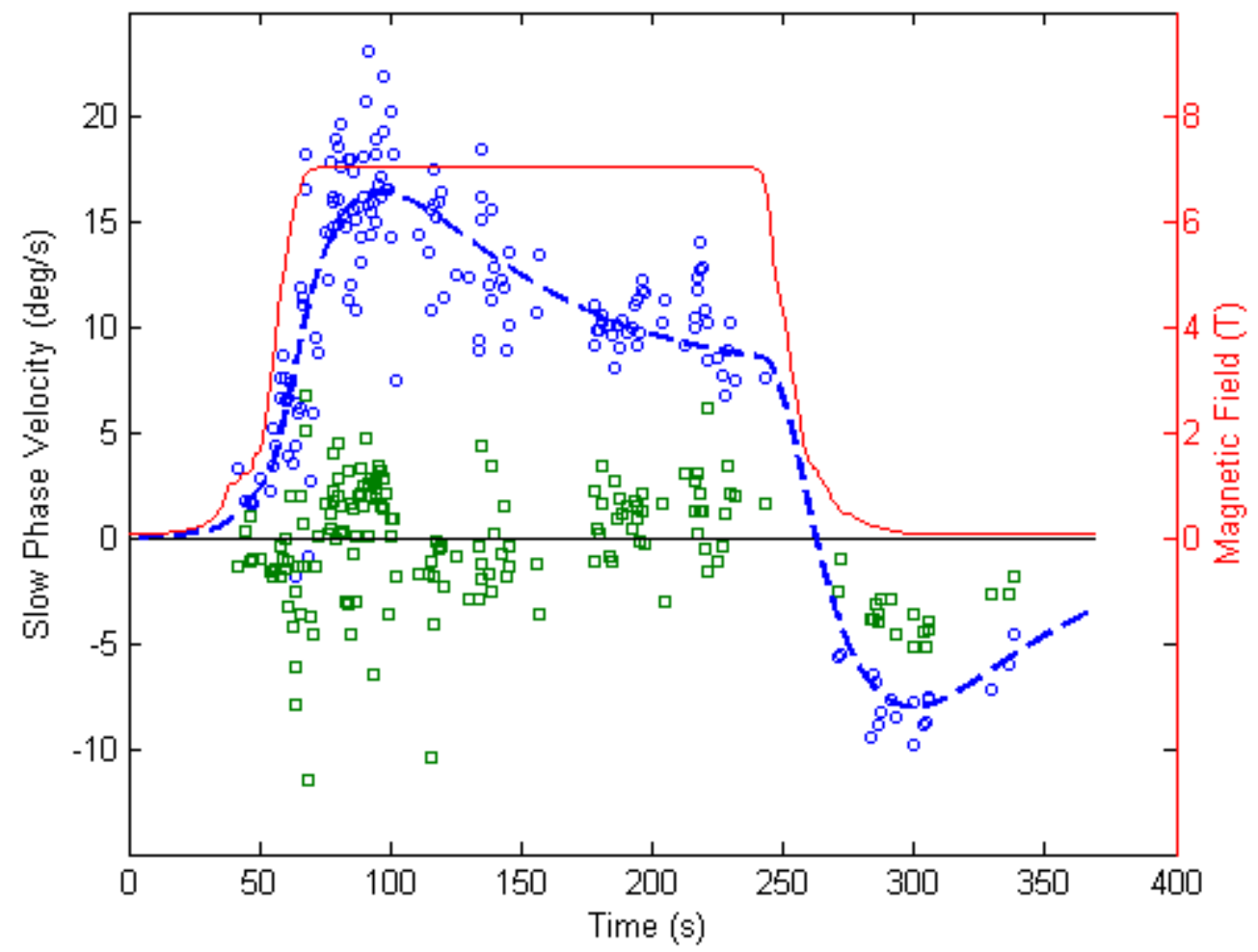

Figure 4: Typical slow phase velocity (SPV) record (subject S023_3_1) showing horizontal (blue circles) and vertical (green squares) components (left-hand scale) together with the time dependence of the magnetic field (solid red line and right-hand scale). The four parameter model fit to the horizontal SPV data points is shown (dashed blue line). Positive horizontal SPV = leftward. Positive vertical SPV = upward. 


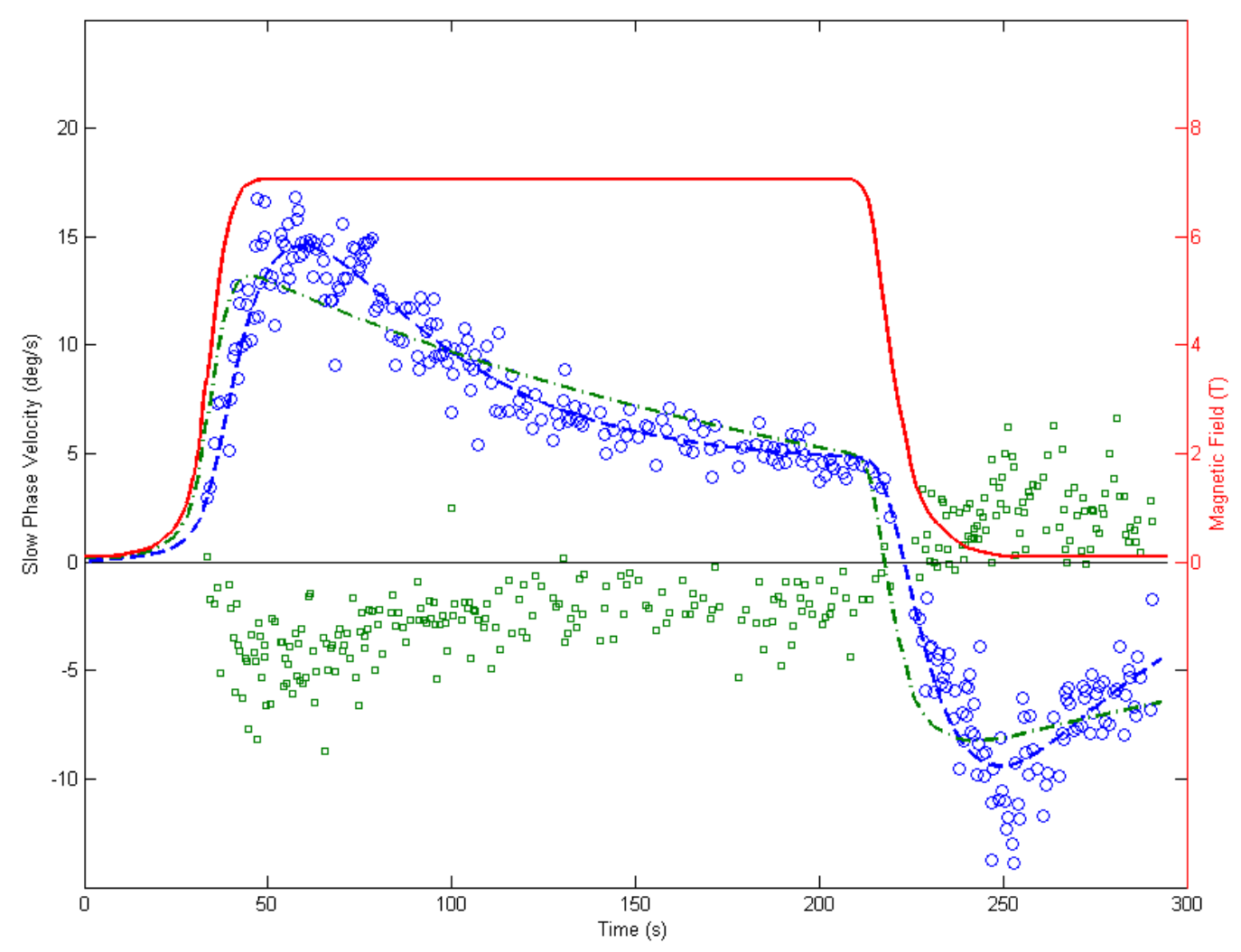

Figure 5: Three parameter fit to S004_3_1 slow phase velocity data (dashed blue line) and fit without cupula dynamic model transfer function (green dashed-dotted line). Otherwise legend is as Figure 4. Parameters for three parameter fit are given in Table 2. Fitted parameters for the model with no cupula dynamics are: $\tau_{L}=0 \mathrm{~s}, \tau_{A}=185 \pm 77 \mathrm{~s}, k_{l}=2.0 \pm 0.1^{\circ} \mathrm{s}^{-1} \mathrm{~T}^{-1}$ and $k_{2}$ $=1.0 \pm 0.2$ 


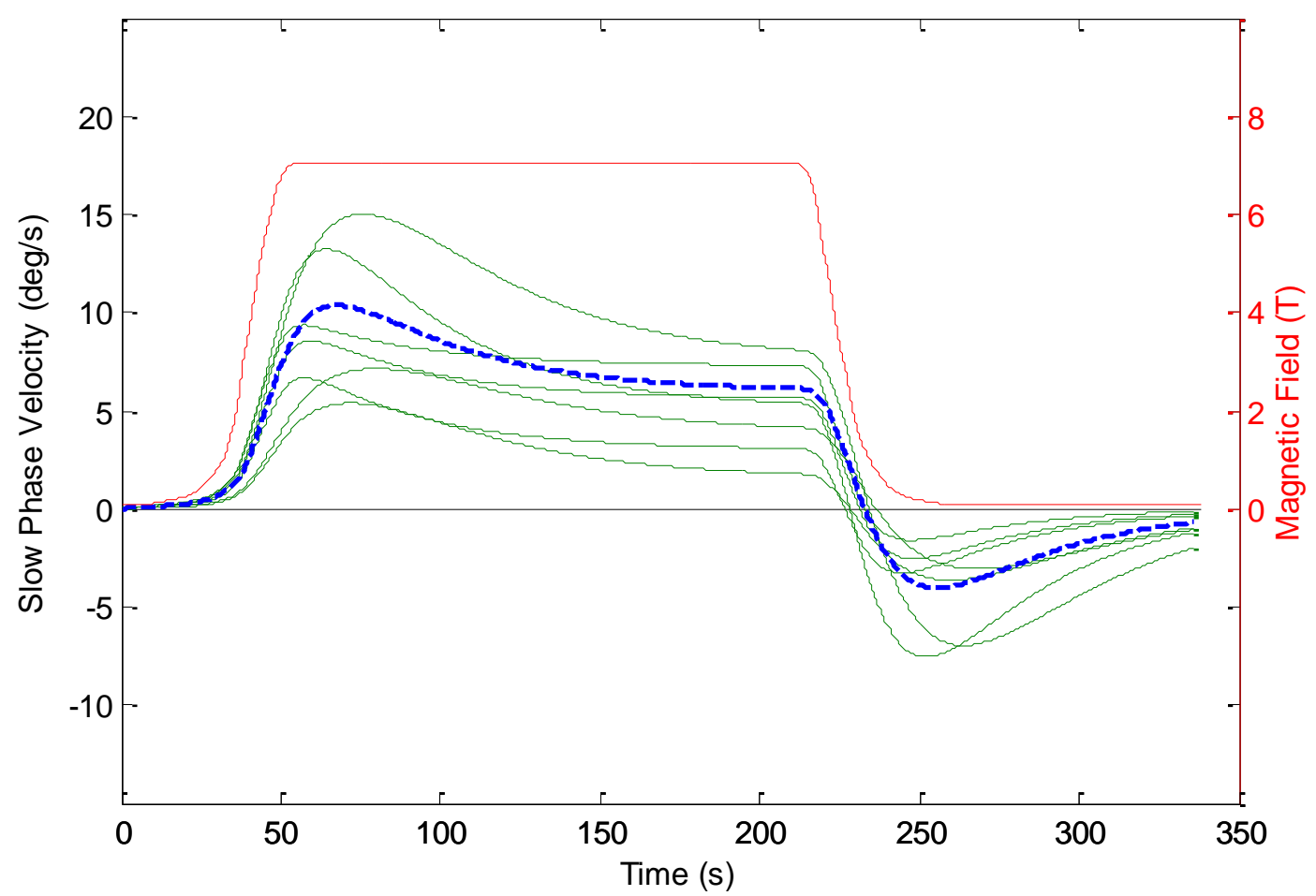

Figure 6: A family of curves describing the predicted SPV response for the magnetic field profile shown. The green solid lines show the 4 parameter fits as given in table 1 . The blue dotted line shows the average fit parameters. 


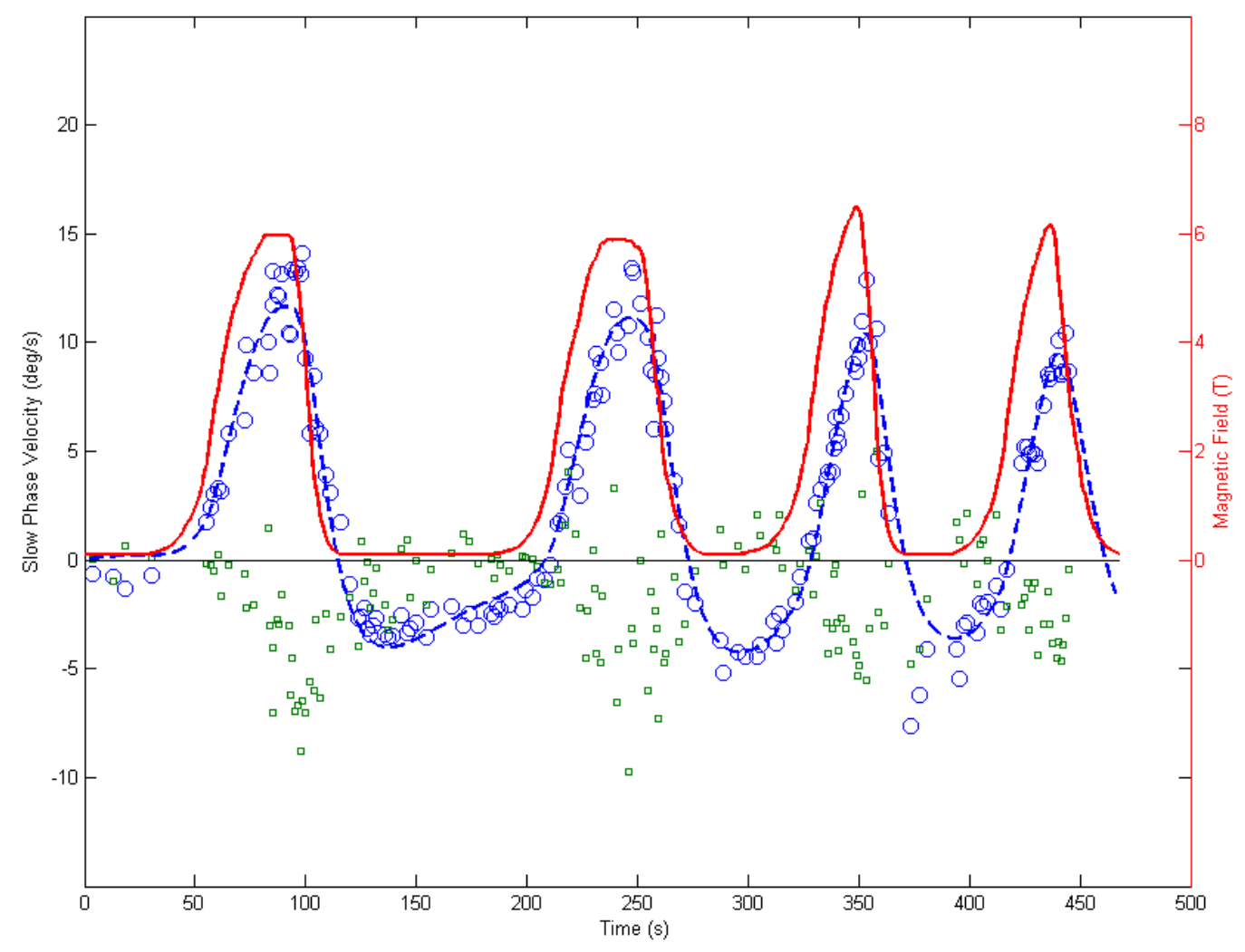

Figure 7: S009_1_1 SPV data fitted using 3 parameters showing good agreement with cupula deflection and adaption model during 4 short exposures to high magnetic field. $\tau_{L}=14.7 \pm$ $1.3 \mathrm{~s}, \tau_{A}=39.3 \mathrm{~s}$ (constrained), $k_{1}=3.4 \pm 0.2^{\circ} \mathrm{s}^{-1} \mathrm{~T}^{-1}$ and $k_{2}=0.80 \pm 0.04-$. The legend is as Figure 4. 

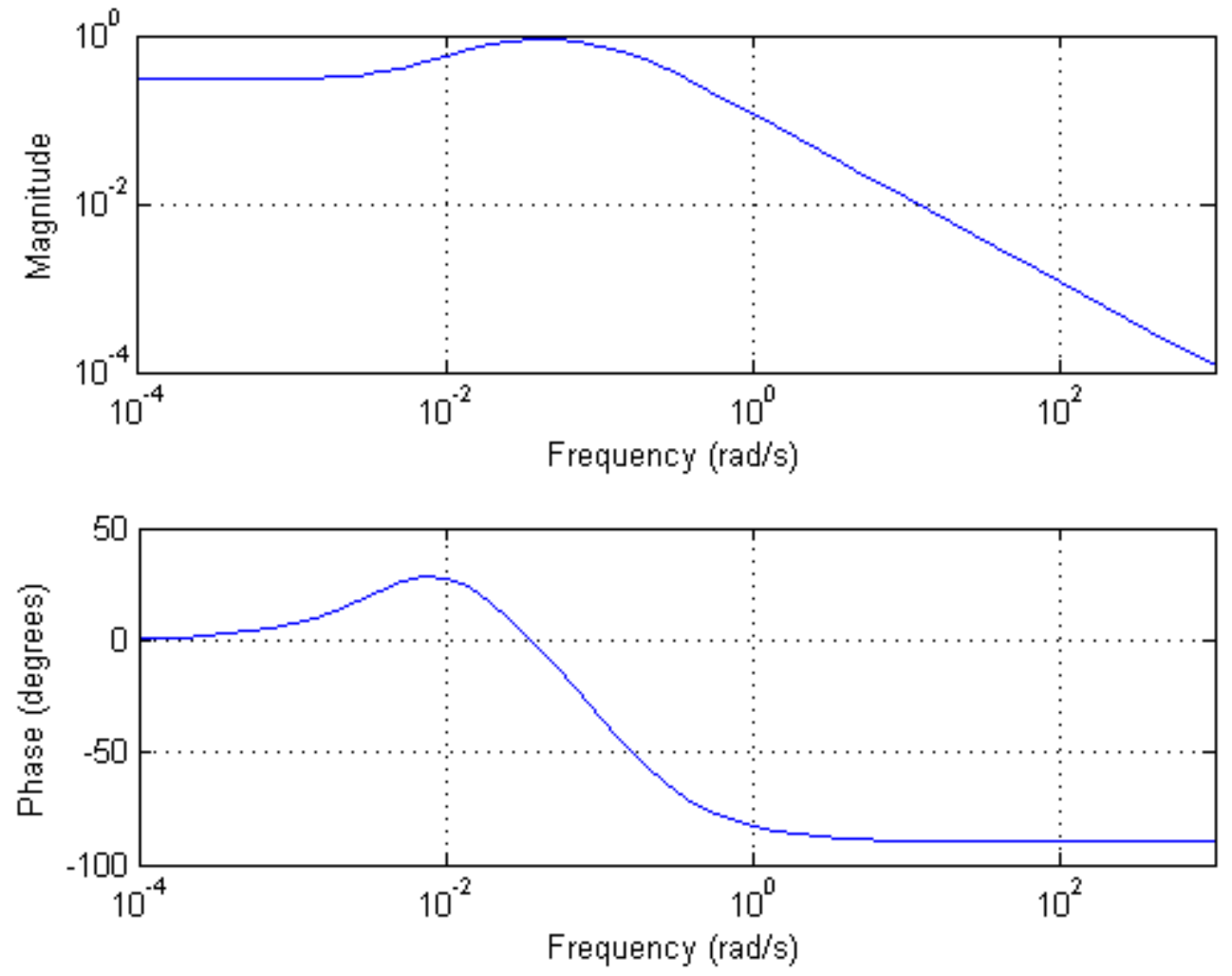

Figure 8: Frequency response of transfer function model given in equation 1 using average fit parameters from Table 2. 


\section{Tables}

Table 1: Four parameter fits for long entry experiment. Uncertainty values (e.g. $\left.\Delta \tau_{A}\right)$ for fitted parameters are given at a $95 \%$ confidence level. Note that multiple trials from subject S004 are averaged first before combining to give final average

\begin{tabular}{|c|c|c|c|c|c|c|c|c|c|}
\hline Subject & $\begin{array}{r}\tau_{L} \\
(s)\end{array}$ & $\begin{array}{c}\Delta \tau_{L} \\
(\mathbf{s})\end{array}$ & $\begin{array}{l}\tau_{A} \\
(\mathbf{s}) \\
\end{array}$ & $\begin{array}{c}\Delta \tau_{A} \\
\text { (s) }\end{array}$ & 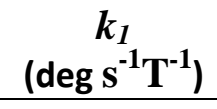 & 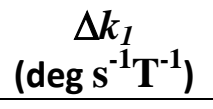 & $k_{2}$ & $\Delta k_{2}$ & MSE \\
\hline S004_3_1 & 14.5 & 4.1 & 35.3 & 10.5 & 3.64 & 0.75 & 0.82 & 0.03 & 2.15 \\
\hline S004_3_6 & 10.0 & 3.3 & 28.4 & 9.3 & 2.73 & 0.57 & 0.72 & 0.05 & 2.00 \\
\hline S004_4_5 & 8.1 & 2.2 & 52.4 & 17.8 & 2.30 & 0.29 & 0.71 & 0.03 & 1.45 \\
\hline average of $\mathrm{S004}$ & 10.9 & & 38.7 & & 2.9 & & 0.75 & & \\
\hline S012_3_6 & 2.8 & 2.9 & 52.3 & 80.2 & 1.49 & 0.35 & 0.31 & 0.12 & 1.97 \\
\hline S016_3_1 & 4.8 & 2.5 & 33.3 & 13.6 & 1.25 & 0.23 & 0.66 & 0.05 & 0.73 \\
\hline S017_3_5 & 23.4 & 4.3 & 28.5 & 11.3 & 1.84 & 0.25 & 0.70 & 0.10 & 1.93 \\
\hline S021_3_1 & 5.1 & 5.9 & 27.8 & 36.7 & 1.49 & 0.74 & 0.47 & 0.23 & 2.06 \\
\hline S023_3_1 & 20.0 & 14.9 & 55.1 & 58.3 & 3.72 & 2.03 & 0.71 & 0.10 & 8.17 \\
\hline
\end{tabular}


Table 2: Three parameter fits for long entry experiment with $\tau_{A}$ constrained to $39.3 \mathrm{~s}$. Note that multiple trials from subjects S004 and S012 are averaged first before combining to give final average and SEM values. The tabulated values for Standard Error of the Mean (SEM) are for a 95\% confidence. The final column gives the MSE value obtained if $\tau_{L}$ is constrained to $0 \mathrm{~s}$ (i.e. no cupula low-pass effect) and $\tau_{A}$ becomes a fitted parameter.

\begin{tabular}{|c|c|c|c|c|c|c|c|c|c|}
\hline Subject & $\begin{array}{l}\tau_{L} \\
(s)\end{array}$ & $\begin{array}{l}\Delta \tau_{L} \\
\text { (s) }\end{array}$ & $\begin{array}{l}\tau_{A} \\
(\mathbf{s})\end{array}$ & 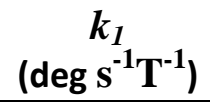 & 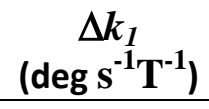 & $k_{2}$ & $\Delta k_{2}$ & MSE & $\begin{array}{c}\text { MSE } \\
\left(\tau_{L}=0 \mathrm{~s}\right)\end{array}$ \\
\hline S004_3_1 & 13.2 & 1.1 & 39.3 & 3.39 & 0.09 & 0.81 & 0.01 & 2.14 & 4.49 \\
\hline S004_4_5 & 10.3 & 1.4 & 39.3 & 2.61 & 0.11 & 0.72 & 0.03 & 1.47 & 2.25 \\
\hline average of 004 & 10.4 & & 39.3 & 2.76 & & 0.74 & & & \\
\hline S009_4_6 & 26.9 & 6.2 & 39.3 & 3.68 & 0.46 & 1.02 & 0.05 & 1.84 & 5.04 \\
\hline average for 012 & 7.1 & & 39.3 & 2.0 & & 0.57 & & & \\
\hline S016_3_1 & 4.1 & 1.6 & 39.3 & 1.2 & 0.1 & 0.65 & 0.0 & 0.7 & 0.9 \\
\hline S017_3_5 & 17.9 & 7.1 & 39.3 & 1.46 & 0.26 & 0.63 & 0.10 & 1.92 & 2.34 \\
\hline S021_3_1 & 3.9 & 3.2 & 39.3 & 1.34 & 0.20 & 0.43 & 0.13 & 2.04 & 2.27 \\
\hline S023_3_1 & 25.5 & 6.3 & 39.3 & 4.57 & 0.57 & 0.75 & 0.06 & 8.13 & 21.30 \\
\hline
\end{tabular}


Table 3: Three parameter fits for short entry experiment with $\tau_{A}$ constrained to $39.3 \mathrm{~s}$. Uncertainty values (e.g. $\Delta \tau_{L}$ ) for fitted parameters are given at a 95\% confidence level.

\begin{tabular}{|c|c|c|c|c|c|c|c|c|}
\hline Subject & $\begin{array}{r}\tau_{L} \\
(\mathrm{~s}) \\
\end{array}$ & $\begin{array}{c}\Delta \tau_{L} \\
(\mathrm{~s})\end{array}$ & $\begin{array}{r}\tau_{A} \\
(s) \\
\end{array}$ & $\begin{array}{c}k_{1} \\
\left(\operatorname{deg~S^{-1}} \mathbf{T}^{-1}\right)\end{array}$ & $\begin{array}{c}\Delta k_{1} \\
\left(\operatorname{deg~} \mathrm{s}^{-1} \mathbf{T}^{-1}\right)\end{array}$ & $k_{2}$ & $\Delta k_{2}$ & MSE \\
\hline S001_1_1 & 14.7 & 6.97 & 39.3 & 1.55 & 0.44 & 0.72 & 0.23 & 2.80 \\
\hline S003_1_1 & 20.5 & 3.65 & 39.3 & 2.71 & 0.34 & 0.87 & 0.09 & 1.36 \\
\hline S004_1_1 & 12.1 & 2.87 & 39.3 & 3.80 & 0.38 & 0.66 & 0.08 & 8.61 \\
\hline S008_1_1 & 9.1 & 1.53 & 39.3 & 2.92 & 0.21 & 0.63 & 0.07 & 2.76 \\
\hline S009_1_1 & 14.7 & 1.34 & 39.3 & 3.43 & 0.19 & 0.80 & 0.04 & 1.71 \\
\hline S012_1_1 & 9.9 & 2.44 & 39.3 & 2.02 & 0.24 & 0.69 & 0.11 & 3.59 \\
\hline S013_1_1 & 9.3 & 2.01 & 39.3 & 4.29 & 0.41 & 0.39 & 0.12 & 3.44 \\
\hline S014_1_1 & 17.5 & 3.13 & 39.3 & 1.77 & 0.18 & 0.73 & 0.08 & 0.97 \\
\hline Averages & 13.5 & & 39.3 & 2.8 & & 0.7 & & \\
\hline $2 *$ SEM & 2.9 & & & 0.7 & & 0.1 & & \\
\hline
\end{tabular}

